\title{
The effects of moderate exercise and overtraining on learning and memory, hippocampal inflammatory cytokine levels, and brain oxidative stress markers in rats
}

\author{
Zahra Jahangiri $^{1,2} \cdot$ Zahra Gholamnezhad $^{1,2}\left(\right.$ Mahmoud Hosseini $^{1,3} \cdot$ Farimah Beheshti $^{4,5} \cdot$ Narges Kasraie $^{6}$
}

Received: 17 June 2019 / Accepted: 9 October 2019 / Published online: 22 October 2019

(C) The Physiological Society of Japan and Springer Japan KK, part of Springer Nature 2019

\begin{abstract}
To investigate the exercise intensity effects on rats' memory and learning, animals were divided into control, moderate training (MT), and overtraining (OT) groups. At training last week, learning and memory was assessed using Morris water maze (MWM) and passive avoidance (PA) tests. Finally, the rat's brains were removed for evaluating oxidative stress and inflammatory cytokines. Overtraining impaired animal's performance in MWM and PA tests. In MT group, hippocampal levels of interleukin 1 beta (IL-1 $\beta$ ) and malondialdehyde (MDA) increased, and thiol contents in hippocampal and cortical tissues decreased compared to control. In OT group, tumor necrosis factor $\alpha$, IL- $1 \beta$, and C-reactive protein hippocampal levels increased, MDA and nitric oxide metabolite in hippocampal and cortical tissues increased, thiol contents, catalase and superoxide dismutase activity in hippocampal and cortical tissues decreased compared to control and MT groups. Overtraining might lead to learning and memory impairment by increasing the inflammatory cytokine and oxidative stress markers.
\end{abstract}

Keywords Moderate training $\cdot$ Overtraining $\cdot$ Learning $\cdot$ Memory $\cdot$ Inflammation $\cdot$ Oxidative stress

\section{Introduction}

Electronic supplementary material The online version of this article (https://doi.org/10.1007/s12576-019-00719-z) contains supplementary material, which is available to authorized users.

Zahra Gholamnezhad

gholamnezhadz@mums.ac.ir; gholamnezhadz@yahoo.com

1 Neurogenic Inflammation Research Center, Mashhad University of Medical Sciences, Mashhad, Iran

2 Neurogenic Inflammation Research Center, Department of Physiology, Faculty of Medicine, Mashhad University of Medical Sciences, Mashhad, IR 9177948564, Iran

3 Division of Neurocognitive Sciences, Psychiatry and Behavioral Sciences Research Center, Mashhad University of Medical Sciences, Mashhad, Iran

4 Neuroscience Research Center, Torbat Heydariyeh University of Medical Sciences, Torbat Heydariyeh, Iran

5 Department of Physiology, School of Paramedical Sciences, Torbat Heydariyeh University of Medical Sciences, Torbat Heydariyeh, Iran

6 Rosenberg School of Optometry, University of the Incarnate Word, San Antonio, TX, USA
It has been well documented that there is a relationship between neuroinflammation and neurodegenerative diseases [1]. Overproduction of inflammatory cytokines such as the tumor necrosis factor- $\alpha$ (TNF- $\alpha$ ), interleukin $1 \beta$ (IL-1 $\beta$ ), interleukin 6 (IL-6) in the brain [2,3] and increased levels of C-reactive protein (CRP) in the plasma have been shown to disrupt memory and increase the risk of Alzheimer's disease (AD) $[4,5]$. Also, oxidative stress status which is accompanied by increased levels of reactive oxygen species (ROS) has a detrimental role in neurodegenerative diseases and impairment of learning and memory [6].

During recent years, the incidence of many chronic noncommunicable diseases, including neurodegenerative disease, chronic heart disease, cancer, metabolic syndrome, and diabetes has increased due to physical inactivity and lifestyle changes [7]. Physical exercise has advantageous effects on many physiological systems, including the central nervous system. The positive effects of exercise on cognitive function, neural health and neuronal protection from various brain insults are well reported $[8,9]$. Also, it has been shown that physical exercise serves as a potent non-pharmacological treatment to improve learning and memory and 
reduce the risk of $\mathrm{AD}[10,11]$. Regular physical exercise can decrease the levels of amyloid oligomer that is very important in the pathogenesis of AD [12]. In addition, moderate exercise that does not lead to exhaustion has anti-inflammatory and antioxidant roles. It can decrease inflammatory cytokines such as TNF- $\alpha$, IL- $1 \beta$, IL- 6 and CRP [13, 14], and oxidative damage by increasing the antioxidant activities $[15,16]$. However, different types of exercise may affect brain plasticity based on the nature, severity and the time lag in between exercises as well as type and age of animal [17].

According to the hormesis theory, both lack of physical stress (inactivity) and excessive stress (overtraining) might result in decreased physiological function [18]. Excessive overloading during competitive training in combination with inadequate recovery or other lifestyle stresses can lead to overreaching and possibly overtraining syndrome. Overtraining is manifested by extreme fatigue and underperformance period, which can last several months to several years [19]. In overtraining, the homeostatic balance involving a wide range of hormonal, metabolic, and immunologic factors is altered $[18,20]$. Overload exercise without adequate rest (overtraining) will lead to tissue injury, especially in muscle and joints, and release of trauma-related cytokines, which activate circulating monocytes to produce inflammatory cytokines [21]. It has been demonstrated that high-intensity exercise increases the levels of TNF- $\alpha$, IL- $1 \beta$, IL- 6 and CRP $[22,23]$. Also, some studies have shown that acute exercise and overtraining lead to the production of reactive oxygen and nitrogen species and oxidative stress, while regular exercise can stimulate the antioxidant system and protect against the side effects of oxidative damage [24]. Overtraining might cause signs and symptoms of cognitive disturbance as well as immunosuppression and inflammation [25].

Some previous studies have shown the beneficial effects of exercise on memory and cognitive processes, while a few studies highlight the effect of type and the training load of exercise on memory, and the probable related mechanisms including inflammation and imbalance of the immune system. However, the effects of overtraining in athletes on brain inflammation and cognition are still under question. Therefore, we decided to investigate the effects of overtraining and moderate exercise on learning and memory function, hippocampal cytokine levels and brain tissues oxidative damage in rats.

\section{Materials and methods}

\section{Animals}

Twenty-one male Wistar rats (6-8 weeks old and weighing 150-200 g) were obtained from the animal house of Mashhad University of Medical Sciences, Mashhad, Iran. Animals were caged in standard controlled conditions (temperature $\left(22 \pm 2{ }^{\circ} \mathrm{C}\right)$ and $12 \mathrm{~h} \mathrm{light/dark} \mathrm{cycle)} \mathrm{and} \mathrm{provided} \mathrm{food} \mathrm{and}$ water ad libitum throughout the experiment. All experiments were performed in accordance to the standards established by the Committee on Animal Research of Mashhad University of Medical Sciences (IR.MUMS.fm.REC.1396.468). The rats were randomly divided into three groups $(n=7)$ : control, moderate trained (MT), and overtrained (OT). The chemical agents which were used for the biochemical assessments were purchased from Merck Company.

\section{Exercise protocol and animal groups}

The animals experienced 1-week familiarizing ( $10 \mathrm{~min} /$ day for 5 days at speed of $12 \mathrm{~m} / \mathrm{min}$ at $0 \%$ degree inclination) on a motorized treadmill with 4 individual lanes prior to the start of the experiments to eliminate the exercise-induced stress. The animals of the control group were placed on the treadmill to acclimate to the stresses of the treadmill environment. The exercising groups undertook a progressive load of training, 6 days/week, to enhance cardiorespiratory fitness which included a 5-min warm up and a 5-min cooldown in each session. The animals of the MT group ran at a speed of 15 meters/min for $30 \mathrm{~min}, 6$ days/week, for 9 weeks (Table 1) [26]. On the other hand, the animals in the OT group were submitted into a 3-phase program. In phase I, the first 4 weeks, the training speed was increased from 15 to $25 \mathrm{~m} / \mathrm{min}$ and the training time from $20 \mathrm{~min}$ to $60 \mathrm{~min}$. In phase II, the second 4 weeks, the training load was kept constant. And in phase III, which constituted the last 3 weeks, the running intensity and the training duration remained unchanged, while the recovery time between the training sessions was decreased from $24 \mathrm{~h}$ to 4,3 and $2 \mathrm{~h}$, and the number of exercise bouts was increased from 1 to 4 in each day (Table 2) [27]. The training programs were evaluated by a performance test at different times.

\section{Performance test}

In this study, the performance test for the animals of the MT group was performed before the start of the main exercise, at the end of the 2nd, 4th and 9th weeks, while for the animals in the OT group, it was performed before the start of the

Table 1 Exercise protocol of the moderate trained (MT) animals

\begin{tabular}{lllll}
\hline Week & $\begin{array}{l}\text { Training } \\
\text { velocity }(\mathrm{m} / \\
\text { min) }\end{array}$ & $\begin{array}{l}\text { Train- } \\
\text { ing time } \\
\text { (min) }\end{array}$ & $\begin{array}{l}\text { Training ses- } \\
\text { sion number } \\
\text { (per day) }\end{array}$ & $\begin{array}{l}\text { Recovery } \\
\text { between exercise } \\
\text { sessions (h) }\end{array}$ \\
\hline 0 & 12 & 10 & 1 & 24 \\
1 & 12 & 20 & 1 & 24 \\
$2-9$ & 15 & 30 & 1 & 24 \\
\hline
\end{tabular}


Table 2 Exercise protocol of the overtrained (OT) animals

\begin{tabular}{llllll}
\hline Week & $\begin{array}{l}\text { Training } \\
\text { phase }\end{array}$ & $\begin{array}{l}\text { Training velocity } \\
(\mathrm{m} / \mathrm{min})\end{array}$ & $\begin{array}{l}\text { Training time } \\
(\mathrm{min})\end{array}$ & $\begin{array}{l}\text { Training session } \\
\text { number (per day) }\end{array}$ & $\begin{array}{l}\text { Recovery between } \\
\text { exercise sessions (h) }\end{array}$ \\
\hline 0 & 1 & 12 & 10 & 1 & 24 \\
1 & 1 & 15 & 20 & 1 & 24 \\
2 & 1 & 20 & 30 & 1 & 24 \\
3 & 1 & 22.5 & 45 & 1 & 24 \\
4 & 1 & 25 & 60 & 1 & 24 \\
$5-8$ & 2 & 25 & 60 & 1 & 24 \\
9 & 3 & 25 & 60 & 2 & 4 \\
10 & 3 & 25 & 60 & 3 & 3 \\
11 & 3 & 25 & 60 & 4 & 2 \\
\hline
\end{tabular}

main exercise, at the end of the $2 \mathrm{nd}, 4 \mathrm{th}, 8 \mathrm{th}, 9 \mathrm{th}, 10 \mathrm{th}$ and 11 th weeks. These performance tests were performed $48 \mathrm{~h}$ after the last training session. The animals were running on the treadmill at a $0 \%$ inclination, at an initial speed of $12 \mathrm{~m} /$ $\mathrm{min}$. The treadmill speed was increased $1 \mathrm{~m} / \mathrm{min}$ every $2 \mathrm{~min}$ until it reached a speed of $20 \mathrm{~m} / \mathrm{min}$. Then, the speed was further increased to $2 \mathrm{~m} / \mathrm{min}$ every $3 \mathrm{~min}$ until exhaustion occurred, which was defined as the time at which the animals touched the shock grid 5 times/min. The body weight of the animals was recorded before each performance test. The performance test $(\mathrm{Pr})$ was calculated according to the following formula:

$\operatorname{Pr}=\Sigma \underset{i}{\operatorname{Pr}}=\Sigma m V_{i} T_{i}=\Sigma m D_{i}=m D(\mathrm{~kg} / \mathrm{m})$

Pr represents the rat's performance expressed in kilogram-meters $(\mathrm{kg} / \mathrm{m}) ; \mathrm{Pr}_{i}$ is the rat's performance during each stage; $\mathrm{m}$ is the body mass; $V_{i}$ is the stage velocity; $T_{i}$ is the stage running time; $D_{i}$ is the stage distance; and $D$ is the total distance covered by the rat [27].

\section{Behavioral tests}

We evaluated the learning and memory by performing the Morris water maze (MWM) and the passive avoidance test (PA) in the last week of training. All behavioral tests were conducted in a noiseless room (between 10:00 and 14:00), under moderate illumination, and the rats were kept in the room for at least $1 \mathrm{~h}$ before testing.

\section{MWM test}

The MWM task consisted of a black circular swimming pool filled with $23-24{ }^{\circ} \mathrm{C}$ water (pool dimensions: $60 \mathrm{~cm}$ deep $\times 136 \mathrm{~cm}$ diameter) that was divided into four equal quadrants with boundaries labeled north (N), east (E), south (S) and west (W). A circular platform $(10 \mathrm{~cm}$ diameter, $28 \mathrm{~cm}$ high) was submerged in the central area of the southeast quadrant of the MWM pool that was hidden about $2 \mathrm{~cm}$ below the water surface. Fixed visual cues at several locations around the maze and on walls of the room determined the navigation path. The tests were recorded with a video camera placed above the center of the pool and connected to a recording system. The animals could swim freely to find the platform during the allotted $60 \mathrm{~s}$, while allowing them to remain on the platform for $15 \mathrm{~s}$. The time latency and the traveled distances to reach the platform were recorded as a measure of spatial learning. If the animals were not able to find the platform after $60 \mathrm{~s}$ of swimming, they were directed to the platform by the examiner and were allowed to stay on it for $15 \mathrm{~s}$. The experiments were repeated with four trials each day for four consecutive days. After $24 \mathrm{~h}$, the hidden platform was removed, and a probe test was carried out to assess spatial memory. The time spent and the traveled path in the target quadrant $\left(\mathrm{Q}_{4}\right)$ were compared between groups [28].

\section{PA test}

The PA task consisted of a light and a dark compartment which were connected by a guillotine door. Before starting the training sessions, the animals were placed into the apparatus and allowed to move freely between the two compartments for $300 \mathrm{~s}$ during two consecutive days. In the following day, the rats were placed in the light compartment, but once they had entered the dark compartment the guillotine door was shut and an electrical shock (2 mA, $2 \mathrm{sec})$ was delivered to the animal's feet. The test phase was conducted at 2, 24 and $48 \mathrm{~h}$ after the training sessions, then the animals were again placed into the light compartment and their latency to enter the dark compartment and the time spent in each compartment per animal subject were measured [29].

\section{Biochemical assessments}

After the behavioral tests, the rats were euthanized under deep anesthesia and their hippocampal and cortical tissues were removed, and the tissues were homogenized with 
phosphate buffer solution (PBS) ( $\mathrm{pH}$ 7.4). The homogenates were then submitted for biochemical assessments and the content of total thiol (SH), malondialdehyde (MDA), superoxide dismutase (SOD), catalase (CAT) and NO metabolite $\left(\mathrm{NO}^{2-}\right)$ was measured in both the hippocampal and cortical tissues, while the TNF- $\alpha$, IL- $1 \beta$ and CRP concentrations were only determined in the hippocampal tissues.

\section{Measurement of TNF- $\alpha$, IL-1 $\beta$ and CRP}

The hippocampal levels of TNF- $\alpha$, IL- $1 \beta$ and CRP measurement were performed with specific rat ELISA kits (TNF- $\alpha$ : Diaclone Co, France) (IL-1 $\beta$, CRP: Zellbio Co, Germany) while adhering to the manufacturer's instructions. The measured absorbance of the samples in a microplate reader (Biotek, USA) was compared with an established standard curve in the same measurement and the concentrations were calculated.

\section{Measurement of MDA}

MDA is an indicator of lipid peroxidation and was measured based on the MDA reaction with thiobarbituric acid (TBA), which produces a pink complex with a peak absorbance at $535 \mathrm{~nm}[30]$.

\section{Measurement of total thiol content}

Total thiol content was measured by the method of Ellman. SH groups produce a yellow complex which has a peak absorbance at $412 \mathrm{~nm}$ [31].

\section{Measurement of SOD activity}

The SOD activity was measured based on a procedure described by Madesh and Balasubramanian. The procedure involves the production of superoxide through auto-oxidation of pyrogallol and the inhibition of superoxide-dependent reduction of 3-4, 5-dimethyl-thiazol-2-yl -2, 5-diphenyl tetrazolium bromide (MTT) conversion to formazan [32].

\section{Measurement of CAT activity}

The activity of CAT was measured according to the method of Aebi, based on the rate of decomposition of hydrogen peroxide $\left(\mathrm{H}_{2} \mathrm{O}_{2}\right)$ by catalase using a spectrophotometer at $240 \mathrm{~nm}[33]$.

\section{Measurement of NO metabolite}

NO metabolite (nitrite) was measured based on the reaction between Griess reagent, sulfanilamide and naphthylethylenediamine (NED) solutions at $520 \mathrm{~nm}$ [34].

\section{Statistical analysis}

The data were presented as mean \pm standard error of the mean (SEM) using the SPSS 16 software. And the data from the behavioral tests and biochemical assessments were compared using a one-way ANOVA followed by an LSD post hoc comparisons test. Statistical significance was considered at $P<0.05$.

\section{Results}

\section{Performance test}

Our results suggest that the gradual increase in the training load improved the performance in the animals of the MT group, however, in the OT group, although the performance was initially improved, in the third stage of exercise (from the 9th week), the performance significantly declined $(P<0.001)$ (Table 3).

\section{Behavioral results}

\section{MWM test}

In this section, the results of the MWM test were presented for two parameters: the time latency and the traveled distance to reach the platform during a 4-day training, and the time spent and the traveled distance in the target quadrant $\left(\mathrm{Q}_{4}\right)$ in the probe day when the platform was removed.

The results showed that during the 4 days, the time latency and the traveled distance to reach the platform in the animals of the OT group were significantly higher than the control group $(P<0.01-P<0.001)$. However, there were no significant differences between these parameters in the MT group compared to the control group. Also, the time

Table 3 Performance (Pr, Kg.m) of the MT (moderate trained) and the OT (overtrained) groups in different weeks

\begin{tabular}{lllc}
\hline Week & Pr & MT & OT \\
\hline 0 & 1 & $65.7 \pm 3.9$ & $64.7 \pm 7.9$ \\
2 & 2 & $84.7 \pm 7.5^{* *}$ & $122.1 \pm 19.7^{* *}$ \\
4 & 3 & $112.2 \pm 6.1^{* * *}$ & $175.5 \pm 30.13^{* *}$ \\
8 & 4 & $140.4 \pm 10.6^{* * *}$ & $210 \pm 32.3^{* *}$ \\
9 & 5 & $138.2 \pm 6.5^{* * *}$ & $127.6 \pm 30.4^{+++}$ \\
10 & 6 & - & $86 \pm 16.5^{+++}$ \\
11 & 7 & - & $62.7 \pm 11.4^{+++}$ \\
\hline
\end{tabular}

The data are presented as mean \pm standard error of the mean $(n=7$ in each group). The data of week zero represent the baseline measurements for each group. $* * P<0.01$ and $* * * P<0.001$ comparison of the zero week and the 2nd, 4th and 8th weeks and ${ }^{+++} P<0.001$ comparison of the 8 th week and the 9th, 10th and 11th weeks 
latency and the traveled distance to reach the platform of the OT group were significantly higher than the MT group $(P<0.01-P<0.001)$ (Fig. 1a, b).

In the probe trial, the animals of the OT group spent less time and traveled a shorter distance in the target quadrant $\left(\mathrm{Q}_{4}\right)$ compared to the control group $(P<0.01)$. There were no significant differences between these parameters in the MT group compared to the control group. Additionally, the time spent and the traveled distance in the $\mathrm{Q}_{4}$ were significantly lower for the OT group compared to the MT group $(P<0.001)$ (Fig. 2a, b).

\section{PA test}

In this section, the results of the PA test including the latency to enter the dark compartment, the time spent in the dark compartment and the time spent in the light compartment at 2, 24 and $48 \mathrm{~h}$ after receiving the electric shock are presented.

There were no significant differences in the latency to enter the dark compartment at 2, 24 and $48 \mathrm{~h}$ after receiving the electric shock of the animals in the MT and OT groups compared to the control group. The latency to enter the dark
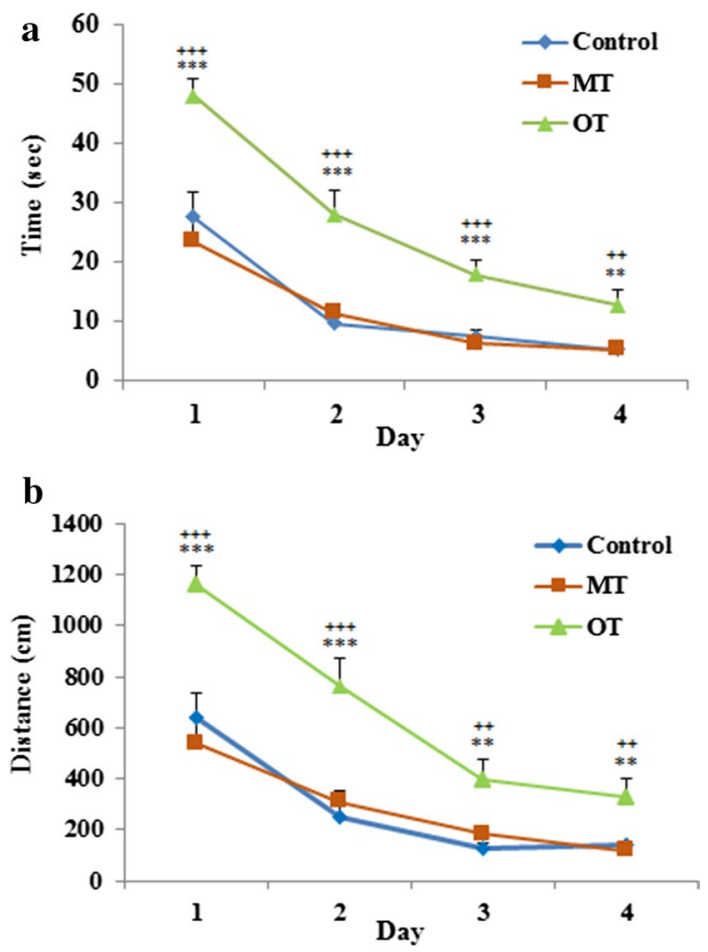

Fig. 1 Comparison of the time latency (a) and the traveled distance (b) to reach the platform in Morris water maze test between the control, MT (moderate trained) and OT (overtrained) groups during the 4 days. The data are presented as mean \pm standard error of the mean ( $n=7$ in each group). $* * P<0.01$ and $* * * P<0.001$ compared to the control group and ${ }^{++} P<0.01$ and ${ }^{+++} P<0.001$ compared to the MT group
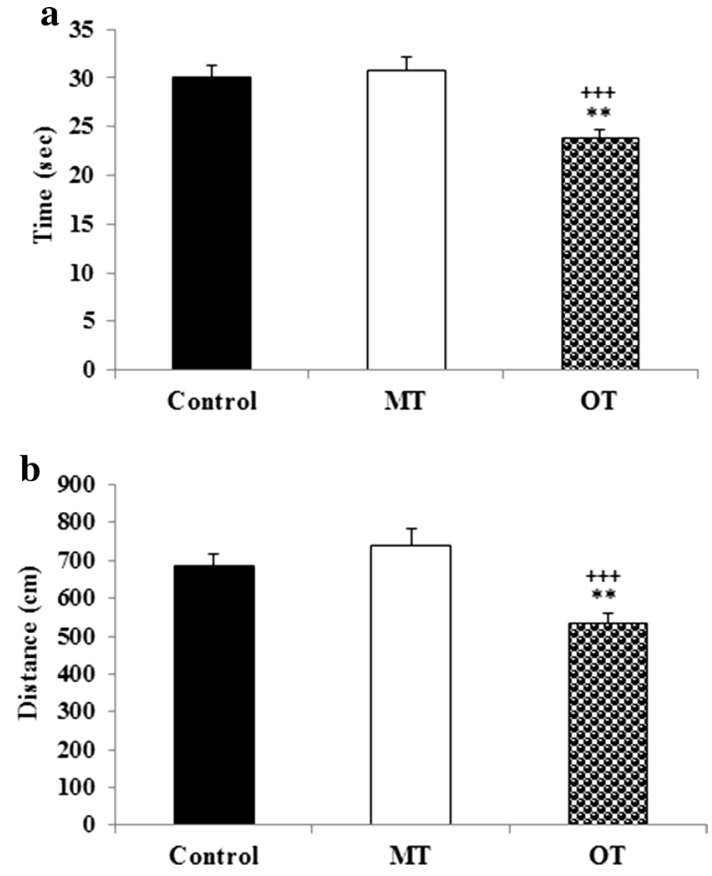

Fig. 2 Comparison of the time spent (a) and the traveled distance (b) in target quadrant between control, MT (moderate trained) and OT (overtrained) groups in probe day. The data are presented as mean \pm standard error of the mean $(n=7$ in each group). $* * P<0.01$ compared to the control group and ${ }^{++} P<0.001$ compared to the MT group

compartment at 2 and $48 \mathrm{~h}$ after receiving the electric shock was significantly lower in the animals in the OT group compared to the MT group $(P<0.05)$ (Fig. 3a).

The time spent in the dark compartment at $48 \mathrm{~h}$ after receiving the electric shock in the OT group was significantly higher than the control group $(P<0.05)$. There were no significant differences in the time spent in the dark compartment by the OT group at 2 and $24 \mathrm{~h}$ after receiving the electric shock and at the 2, 24 and $48 \mathrm{~h}$ after receiving the electric shock for the MT group compared to the control group. Also, in the OT group, the time spent in the dark compartment at 2, 24 and $48 \mathrm{~h}$ after receiving the electric shock was significantly higher than the MT group $(P<0.05-P<0.01)$ (Fig. 3b).

The time spent in the light compartment at $48 \mathrm{~h}$ after receiving the electric shock of the animals in the OT group was significantly lower than the control group $(P<0.05)$. There were no significant differences between the time spent in the light compartment at 2 and $24 \mathrm{~h}$ after receiving the electric shock in the OT group and at 2, 24 and $48 \mathrm{~h}$ after receiving the electric shock in the MT group compared to the control group. Also, in the OT group, the time spent in the light compartment at 2, 24 and $48 \mathrm{~h}$ after receiving the electric shock was significantly lower than the MT group $(P<0.05-P<0.01)$ (Fig. 3c). 

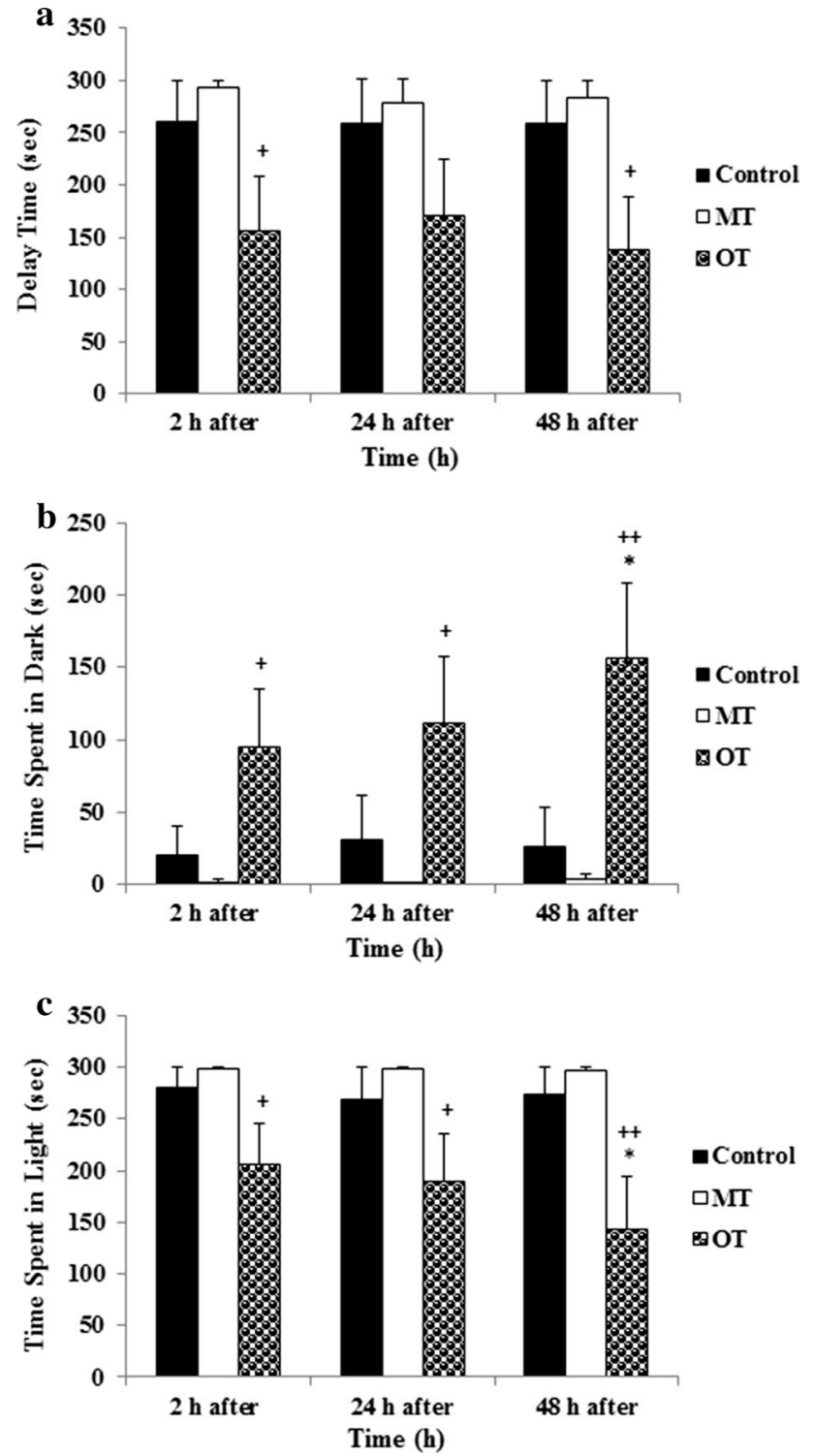

Fig. 3 Comparison of the latency to enter the dark compartment (a), time spent in the dark compartment (b) and the time spent in the light compartment (c) at 2, 24 and $48 \mathrm{~h}$ after receiving the electric shock between the control, MT (moderate trained) and OT (overtrained) groups. The data are presented as mean \pm standard error of the mean ( $n=7$ in each group). $* P<0.05$ compared to the control group and ${ }^{+} P<0.05$ and ${ }^{++} P<0.01$ compared to the MT group

\section{Biochemical results}

\section{Hippocampal cytokine levels}

The hippocampal levels of the TNF- $\alpha$ in the OT group were significantly higher than the control group $(P<0.01)$. There were no significant differences between the TNF- $\alpha$ levels of the MT group compared to the control group. Also, the hippocampal levels of the TNF- $\alpha$ in the OT group were higher than the MT group $(P<0.01)$ (Fig. 4a).
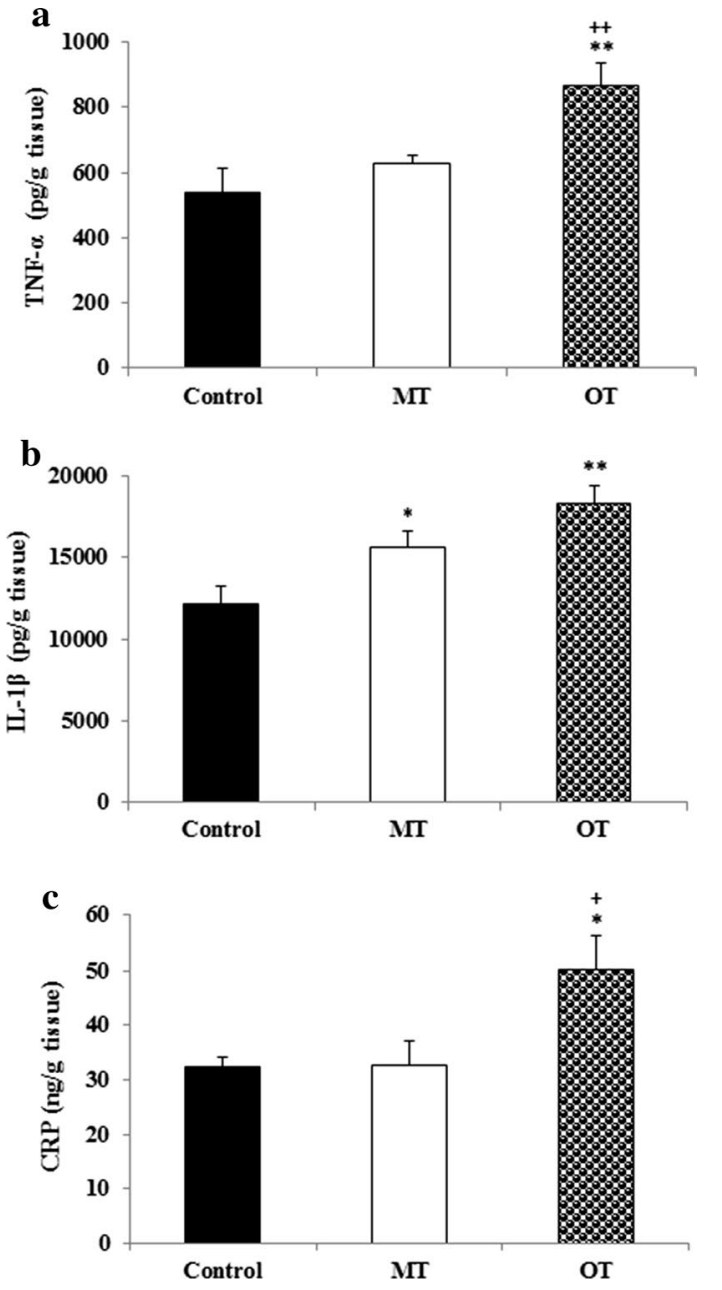

Fig. 4 Comparison of the hippocampal TNF- $\alpha$ (a), IL-1 $\beta$ (b) and CRP (c) levels between the control, MT (moderate trained) and OT (overtrained) groups. The data are presented as mean \pm standard error of the mean ( $n=7$ in each group). $* P<0.05$ and $* * P<0.01$ compared to the control group and ${ }^{+} P<0.05$ and ${ }^{++} P<0.01$ compared to the MT group

The hippocampal levels of the IL- $1 \beta$ in both the MT and OT groups were significantly higher than the control group $(P<0.05-P<0.01)$. There were no significant differences between the IL-1 $\beta$ levels of the OT group compared to the MT group (Fig. 4b).

Our results showed that the hippocampal levels of the CRP in the animals in the OT group were significantly higher than the control group $(P<0.05)$. And there were no significant differences between the CRP levels of the MT group compared to the control group. Also, the hippocampal levels of the CRP in the OT group were higher than the MT group $(P<0.05)($ Fig. $4 \mathrm{c})$. 


\section{Hippocampal stress oxidative markers}

The animals of the MT and OT groups had more MDA concentrations and lower thiol contents in their hippocampal tissues compared to the control group $(P<0.001)$. Additionally, in the hippocampal tissues of the OT group, MDA levels were significantly higher compared to the MT group $(P<0.001)$, however, thiol contents were lower than the MT group $(P<0.001)$ (Fig. 5a, b).

The animals in the OT group had lower CAT and SOD levels in their hippocampal tissues compared to the control group $(P<0.001)$. In the MT group, there were no significant differences between the hippocampal levels of CAT and SOD compared to the control group $(P>0.05)$. Additionally, in the hippocampal tissues of the OT group, CAT and SOD levels were significantly lower than the MT group $(P<0.001)$ (Fig. 5c, d).

\section{Cortical stress oxidative markers}

The animals in the OT group had more MDA concentrations and lower thiol contents in their cortical tissues compared to the control group $(P<0.05-P<0.001)$. In the MT group, there were no significant differences between the cortical
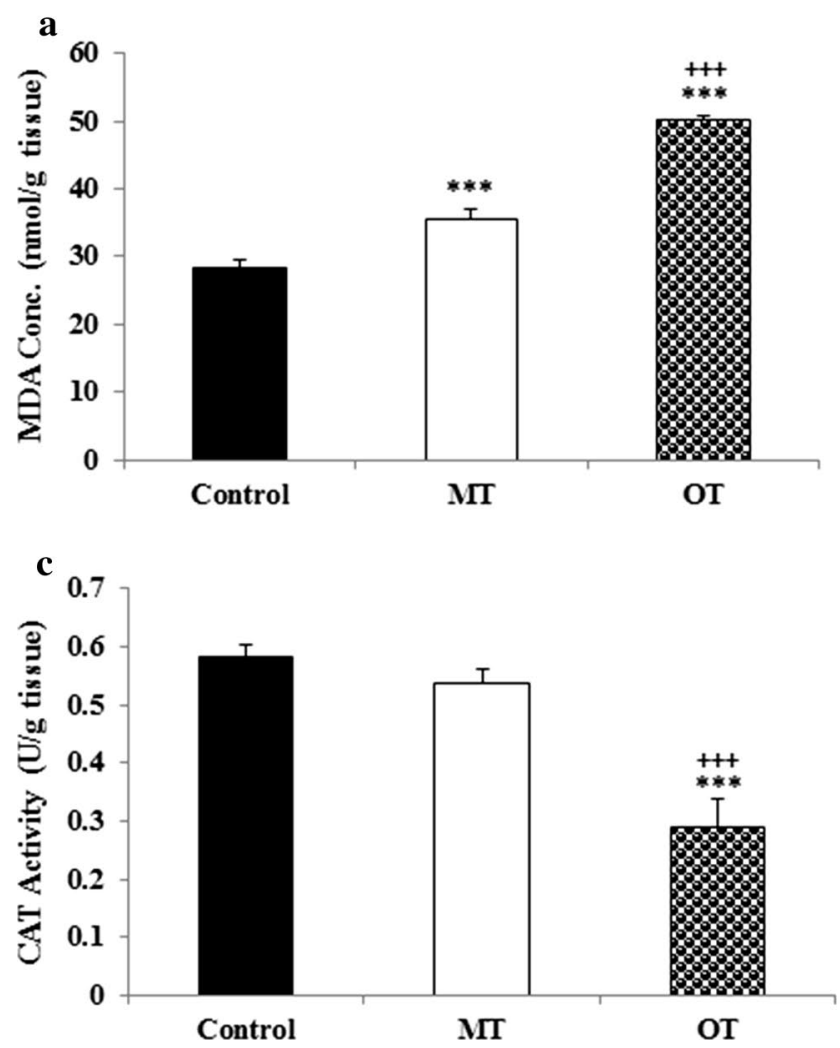

Fig. 5 Comparison of the MDA concentrations (a), thiol contents (b), CAT (c) and SOD (d) levels in the hippocampal tissues of the control, MT (moderate trained) and OT (overtrained) groups. The data are levels of MDA compared to the control group, however, the cortical thiol contents were lower than the control group $(P<0.001)$. Also, the animals of the OT group had more MDA concentrations and lower thiol contents in their cortical tissues compared to the MT group $(P<0.05-P<0.001)$ (Fig. 6a, b).

The animals of the OT group had lower CAT and SOD levels in their cortical tissues compared to the control group $(P<0.001)$. In the MT group, there were no significant differences in the cortical levels of CAT and SOD compared to the control group. Additionally, CAT and SOD levels in the cortical tissues of the OT group were significantly lower than the MT group $(P<0.001)$ (Fig. 6c, d).

\section{NO metabolite}

The NO metabolite concentrations in the hippocampal tissues of the OT group were significantly higher than the control group $(P<0.05)$. However, there were no significant differences between the NO metabolite concentrations of the MT group compared to the control group. Therefore, in the hippocampal tissues of the OT group, the NO metabolite concentrations were higher than the MT group $(P<0.01)$ (Fig. 7a). Additionally, the NO metabolite concentrations in
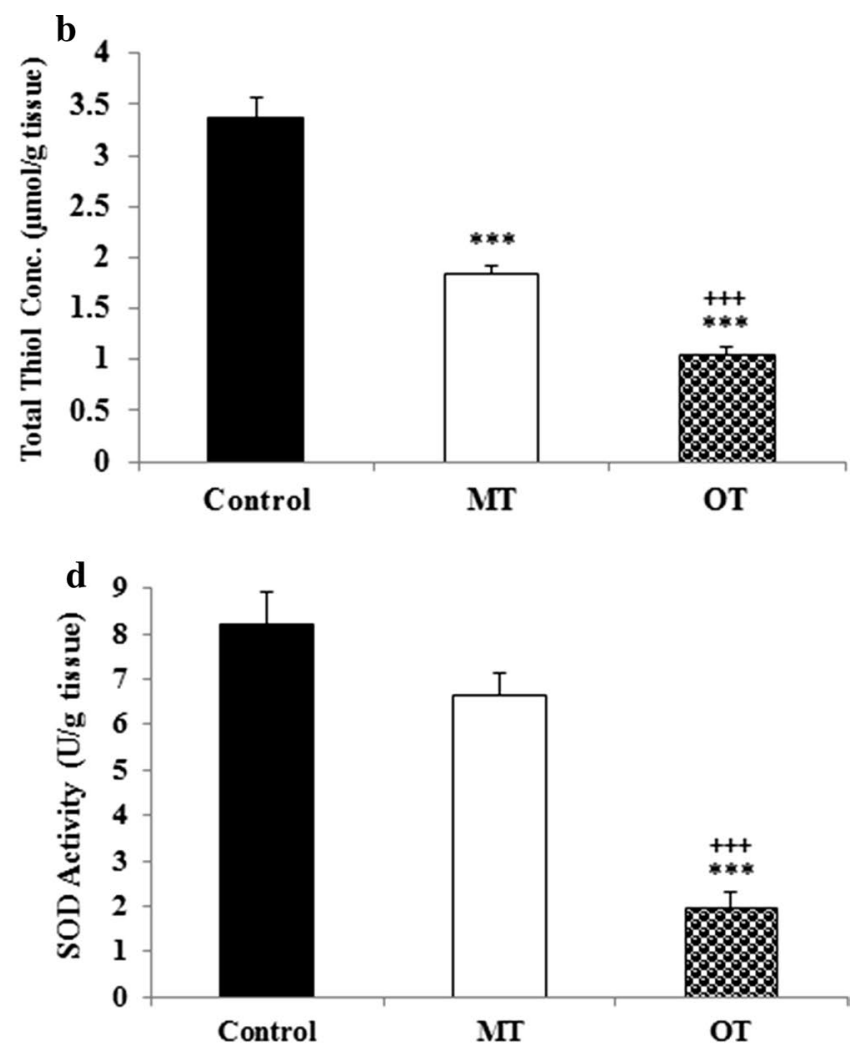

presented as mean \pm standard error of the mean ( $n=7$ in each group). *** $P<0.001$ compared to the control group and ${ }^{+++} P<0.001 \mathrm{com}-$ pared to the MT group 

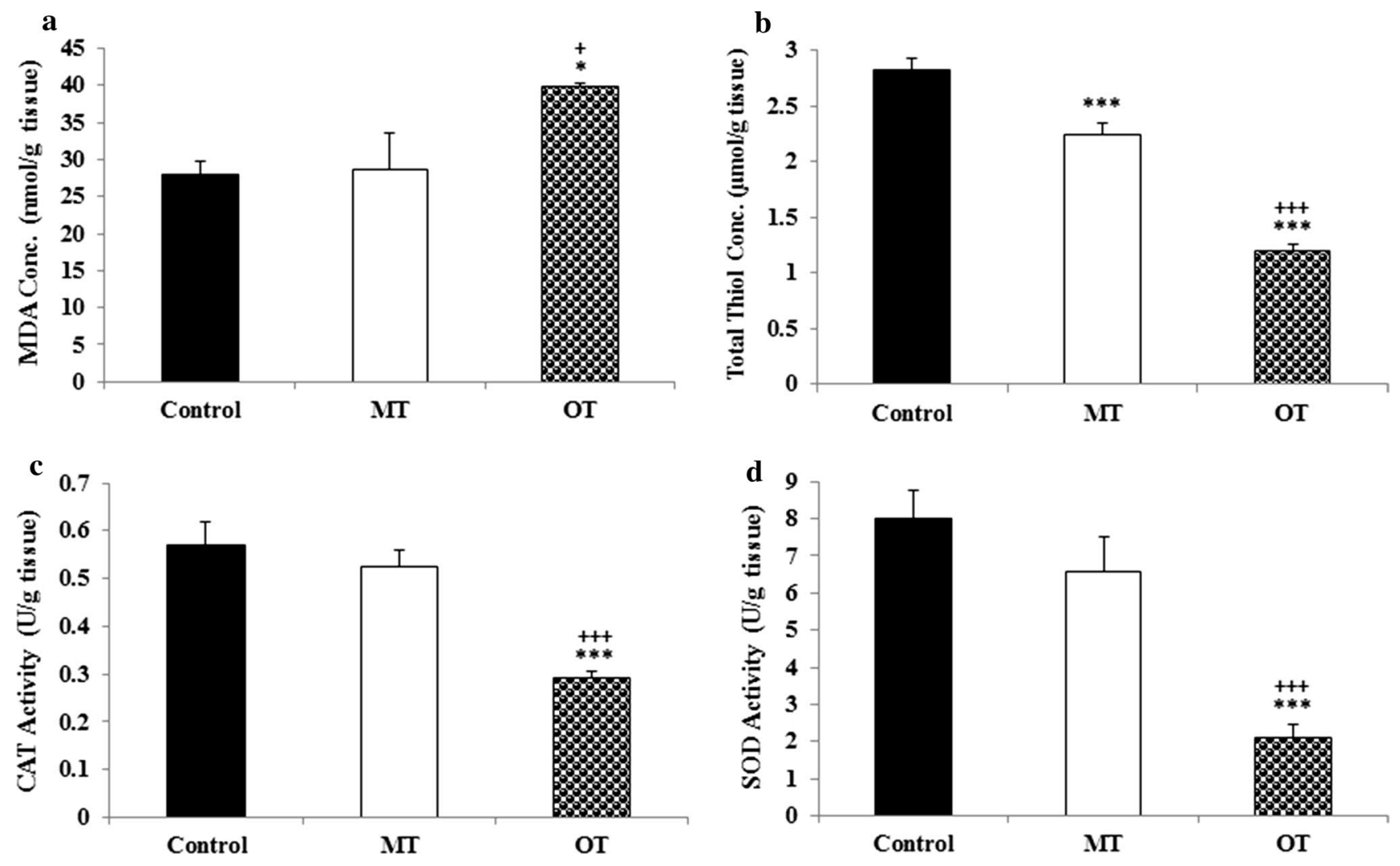

Fig. 6 Comparison of MDA concentrations (a), thiol contents (b), CAT (c) and SOD (d) levels in cortical tissues between the control, MT (moderate trained) and OT (overtrained) groups. The data are

presented as mean \pm standard error of the mean ( $n=7$ in each group). $* P<0.05$ and $* * * P<0.001$ compared to the control group and ${ }^{+} P<0.05$ and ${ }^{+++} P<0.001$ compared to the MT group

the cortical tissues of the OT group were also higher than the control group $(P<0.001)$. While, there were no significant differences between the NO metabolite concentrations of the MT group compared to the control group. Also, in the cortical tissues of the OT group, the NO metabolite concentrations were higher than the MT group $(P<0.001)$ (Fig. 7b).

\section{Discussion}

In this study, the performance test was used as an indicator for measuring the training load. Previous studies have indicated that high-intensity training in combination with inadequate recovery leads to a reduction in performance (non-functional overreaching) and can cause an overtraining syndrome [35]. Our results showed that a gradual increase in the training load improved the performance in the animals in the MT and OT groups in the first two phases of the training ( 8 weeks), while in the third phase of the OT group training, signs and symptoms of overtraining syndrome were observed which included lethargy, fatigue and marked underperformance [27, 36].

The beneficial effects of regular physical activity on the brain function and central nervous system (CNS) adaptations by increasing neurogenesis, synaptic plasticity and long-term potentiation (LTP) in rodents have been previously demonstrated. These biochemical and structural effects may improve performance in behavioral tests, especially learning and memory in rodents [37]. In our study, there were no significant differences between the parameters of the MWM and PA tests of the animals in the MT group compared to the control group. Other studies have also indicated that moderate intensity treadmill exercise $(V=17-20 \mathrm{~m} / \mathrm{min}$, $40 \mathrm{~min} /$ day for 4-12 weeks) has no significant effect on spatial learning (visual and motor functions) in the MWM test $[38,39]$, although the spatial memory was improved in moderately trained animals [39]. Additionally, it has been reported that rats avoidance memory was unaffected by moderate treadmill activity $(V=14-20 \mathrm{~m} / \mathrm{min}, 30-40 \mathrm{~min} /$ day for 4 weeks) $[39,40]$.

Our study results indicated that overtraining induced impairment of spatial learning and memory, and passive avoidance memory in rats. It was shown that that highintensity running exercise $(V=21 \mathrm{~m} / \mathrm{min}, 90 \mathrm{~min} /$ day for 5 weeks) has a negative effect on spatial learning compared 

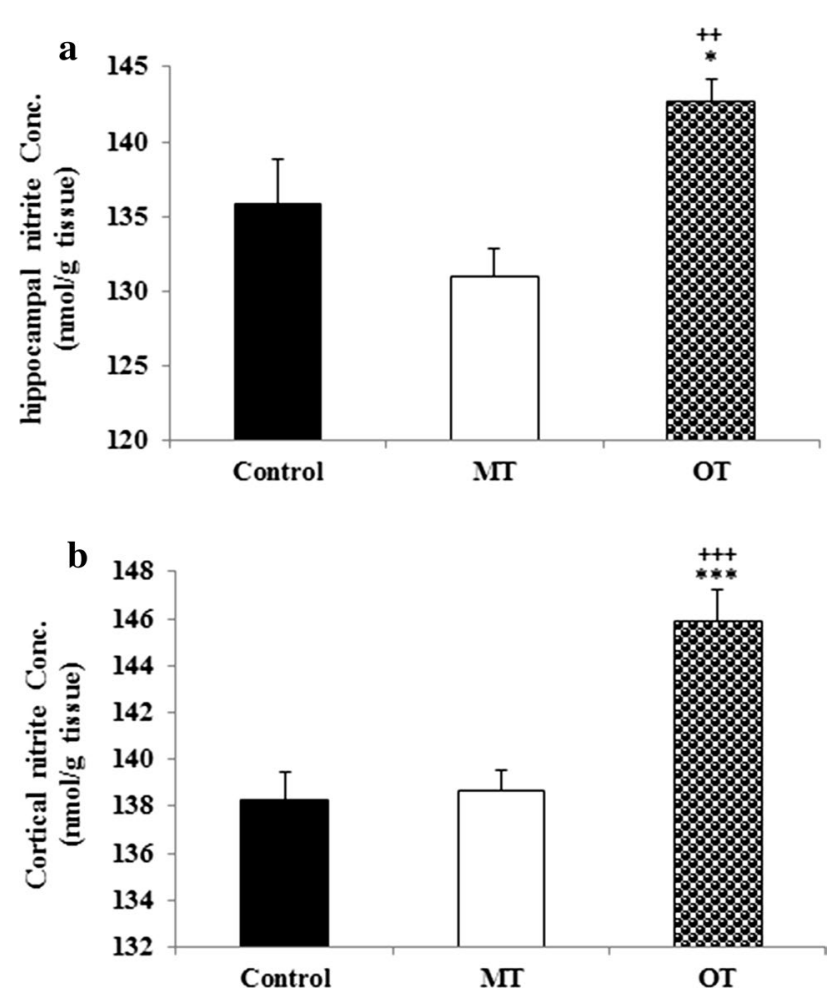

Fig. 7 Comparison of the hippocampal (a) and cortical (b) NO metabolite concentrations between the control, MT (moderate trained) and OT (overtrained) groups. The data are presented as mean \pm standard error of the mean $(n=7$ in each group). $* P<0.05$ and $* * * P<0.001$ compared to the control group and ${ }^{++} P<0.01$ and ${ }^{+++} P<0.001$ compared to the MT group

to the control and low-intensity runner mice, however, both intensities of exercise had no significant effect on spatial memory retention in the MWM test [41]. Few studies have investigated the effect of overtraining on the PA test of intact animals. While some have shown that overtraining $(1 \mathrm{~h} \mathrm{swimming} /$ day for 6 weeks, followed by an increase in the duration to $4.5 \mathrm{~h}$ during the last 2 final weeks) leads to a better performance on the PA test [42], others have reported that strenuous exercise $(V=30 \mathrm{~m} / \mathrm{min}, 40 \mathrm{~min} /$ day for 30 days) has no effect on the outcomes of the PA test [39]. The intensity and duration of the training, as well as the age of animals are important confounding variables impacting the results of some previous studies. The probable mechanisms related to memory and learning modulation effects of these variables have been investigated in this study.

Our results showed that in the animals of the MT group, the hippocampal levels of IL-1 $\beta$ were higher than the control group, however, there were no significant differences between the hippocampal levels of TNF- $\alpha$ and CRP in the MT group compared to the control group. Several studies have reported that mild to moderate intensity of regular physical activity can suppress the expression of inflammatory cytokines and decrease the levels of TNF- $\alpha$, IL- $1 \beta$,
IL-6 and CRP. Balancing the inflammatory cytokine levels may be a sign of physical adaptation to exercise to create an anti-inflammatory environment $[13,14]$. On the other hand, some studies have shown that moderate treadmill exercise ( $V=10-20 \mathrm{~m} / \mathrm{min}, 30-60 \mathrm{~min} /$ day for 5-8 weeks) has no effect on the expression and levels of inflammatory cytokines in the serum and hippocampus [20,43]. Additionally, it was demonstrated that there is a correlation between the severity and duration of exercise training and the immune system's response [44]. IL-1 has a concentration-dependent effect on learning and memory consolidation. At physiologic concentration, IL-1 enhances the LTP and promotes memory, while at high levels it inhibits the LTP and synaptic strengths [45]. The ratio of the IL-1 $\beta$ and its antagonist receptor, the IL-1ra, in the brain has shown to be correlated with brain inflammation and memory impairment [46]. In this study, high levels of hippocampal IL- $1 \beta$ might explain the reason why moderate exercise did not improve spatial memory and passive avoidance results. However, it is worthy to note that in our study the IL-1 $\beta / \mathrm{IL}-1$ ra ratio was not measured.

It has been suggested that an excessive training load without adequate recovery could cause microtrauma and inflammation in muscles and joints, and lead to recruitment of pro-inflammatory cytokines (IL-1 $\beta, \mathrm{TNF} \alpha$, and IL-6) and induce systemic inflammation $[20,35]$. Our results indicated that overtraining increased the levels of certain inflammatory cytokines such as TNF- $\alpha$, IL- $1 \beta$ and CRP in the hippocampal tissues compared to the control group. The hippocampal levels of TNF- $\alpha$ and CRP were also higher than the MT group, while there were no significant differences between the IL-1 $\beta$ levels in the OT group compared to the MT group. It has been suggested that an increase in the levels of TNF- $\alpha$ and IL- $1 \beta$ can lead to the destruction of neurons, especially in the hippocampal region, and therefore, can contribute to learning and memory impairment [47]. It has also been indicated that the stress resulting from high-intensity exercise can impair brain functions [48] through an increase in the levels of hormones and cytokines, such as glucocorticoids and IL-18 which can activate glial cells, especially the microglia $[49,50]$. And the activation of microglia in the hippocampus can lead to an increase in the production of inflammatory cytokines such as TNF- $\alpha$, IL- $1 \beta$, ROS and NO [51]. Similar to our findings, Sun et al. reported that high-intensity treadmill exercise $(V=20 \mathrm{~m} / \mathrm{min}$, duration of fatigue/day for 7 days) activated astrocytes and microglia and increased expression of TNF- $\alpha$ and IL- $1 \beta$ in the hippocampus of rats which led to cognitive impairment [22]. Furthermore, some studies have shown that CRP has a predisposing role in dementia and cognitive impairment, and that high levels of CRP might cause AD [5].

Several studies have indicated that regular physical activity can regulate oxidant/antioxidant balance [52], and activate the antioxidant system against free radicals [53]. In our 
study, the hippocampal levels of MDA were higher in the MT group compared to the control group, while the thiol contents in both the hippocampal and cortical tissues were lower compared to the control group. There were also no significant differences between the cortical levels of MDA, SOD, CAT activities, and NO metabolite concentrations in both the hippocampal and cortical tissues of the MT group compared to the control group. The results of hippocampal and cortical oxidant/antioxidant balance in the MT group are in agreement with the results of behavioral response and increase in levels of IL- $\beta$. It had been reported that moderate treadmill exercise ( $V=15 \mathrm{~m} / \mathrm{min}, 50 \mathrm{~min} /$ day for 8 weeks $)$ attenuates oxidative state of the hippocampus and improves cognitive impairment in the aged rats [54]. As a limitation of this study, the serum and muscle oxidant/antioxidant balance had not been evaluated. However, improvement in animal performance of the MT group despite the elevation of the hippocampal MDA level might propose the modulatory action of ROS in activating physiological response including antioxidant protection, repair of oxidative damage and muscle contraction [53]. A close relationship between ROS production, oxidative damage and overload exercise induced muscle hypoxia has been shown previously as well $[18,55]$. Also, strenuous aerobic exercise causes oxidative damage and lipid peroxidation through an increase in the release of certain hormones such as epinephrine, metabolism of prostanoids, xanthine oxidase, nicotinamide adenine dinucleotide phosphate (NADPH) oxidase and macrophage activity [56]. Following high-intensity aerobic exercises, there is an increase in the levels of ROS, NO and oxidative damage, as well as a decrease in the antioxidant enzymes activity due to the activation of the antioxidant system against free radicals [51, 57]. Our results showed that overtraining led to an increase in the levels of MDA and NO metabolite, and a decline in the thiol content, SOD and CAT activities in both the cortical and hippocampal tissues compared to the control and the MT groups. These results suggest the potential role of oxidative stress in the detrimental effects of overtraining on learning and memory. Similar to our findings, Aguiar et al. also showed that high-intensity treadmill exercise $(V=16.5 \mathrm{~m} / \mathrm{min}, 45 \mathrm{~min} /$ day for 8 weeks) which was above the anaerobic threshold in mice, increased oxidative damage and mitochondrial dysfunction as well as decreased the frontal cortex BDNF level [58]. It was reported that overtraining exercises (1-4.5 h swimming/day) did not induce oxidative damage in the brain [42], however, in this study the underperformance state of animals had not been documented. The biochemical findings of the OT group such as the hippocampal and cortical oxidant/antioxidant balance and inflammatory cytokine levels support the results of the behavioral test which indicated learning and memory impairment.

To the best of our knowledge, a few studies have evaluated the effects of moderate training in intact animals or have compared the level of exercise intensity in normal rats. The positive effects of moderate exercise on different models of learning and memory impairment (e.g., LPS, diabetic) have also been previously shown $[38,59]$. While it is known that the age of animals, training duration and intensity play important roles in exercise neuroendocrine and cognitive effects, further investigations are needed to explain the probable mechanism responsible for the elevation of the IL- $1 \beta$ and MDA concentrations, as well as the unchanged memory and learning scores.

\section{Conclusion}

In conclusion, the result of our study showed that overtraining led to behavioral impairment, increased levels of inflammatory cytokines and oxidative stress markers, and induced learning and memory impairment.

Acknowledgements The results described in this paper are part of the M.Sc. student's thesis. The authors would like to thank the Vice Presidency of Research of Mashhad University of Medical Sciences for financial support.

\section{Compliance with ethical standards}

Conflict of interests The authors declare no conflicts of interest in this study.

\section{References}

1. Yirmiya R, Goshen I (2011) Immune modulation of learning, memory, neural plasticity and neurogenesis. Brain Behav Immun 25(2):181-213. https://doi.org/10.1016/j.bbi.2010.10.015

2. Palin K, Bluthe RM, Verrier D, Tridon V, Dantzer R, Lestage J (2004) Interleukin-1beta mediates the memory impairment associated with a delayed type hypersensitivity response to bacillus Calmette-Guerin in the rat hippocampus. Brain Behav Immun 18(3):223-230. https://doi.org/10.1016/j.bbi.2003.09.002

3. Donzis EJ, Tronson NC (2014) Modulation of learning and memory by cytokines: signaling mechanisms and long term consequences. Neurobiol Learn Mem 115:68-77. https://doi. org/10.1016/j.nlm.2014.08.008

4. Ravaglia G, Forti P, Maioli F, Brunetti N, Martelli M, Talerico T, Bastagli L, Muscari A, Mariani E (2004) Peripheral blood markers of inflammation and functional impairment in elderly community-dwellers. Exp Gerontol 39(9):1415-1422. https://doi. org/10.1016/j.exger.2004.06.010

5. Ravaglia G, Forti P, Maioli F, Chiappelli M, Montesi F, Tumini E, Mariani E, Licastro F, Patterson C (2007) Blood inflammatory markers and risk of dementia: the Conselice study of brain aging. Neurobiol Aging 28(12):1810-1820. https://doi.org/10.1016/j. neurobiolaging.2006.08.012

6. Diniz BS, Mendes-Silva AP, Silva LB, Bertola L, Vieira MC, Ferreira JD, Nicolau M, Bristot G, da Rosa ED, Teixeira AL, Kapczinski $F$ (2018) Oxidative stress markers imbalance in late-life depression. J Psychiatr Res 102:29-33. https://doi.org/10.1016/j. jpsychires.2018.02.023 
7. Lee IM, Shiroma EJ, Lobelo F, Puska P, Blair SN, Katzmarzyk PT, Lancet Physical Activity Series Working G (2012) Effect of physical inactivity on major non-communicable diseases worldwide: an analysis of burden of disease and life expectancy. Lancet 380(9838):219-229. https://doi.org/10.1016/ S0140-6736(12)61031-9

8. Carro E, Trejo JL, Busiguina S, Torres-Aleman I (2001) Circulating insulin-like growth factor I mediates the protective effects of physical exercise against brain insults of different etiology and anatomy. J Neurosci 21(15):5678-5684

9. Tillerson JL, Caudle WM, Reveron ME, Miller GW (2003) Exercise induces behavioral recovery and attenuates neurochemical deficits in rodent models of Parkinson's disease. Neuroscience 119(3):899-911

10. Saadati H, Esmaeili-Mahani S, Esmaeilpour K, Nazeri M, Mazhari S, Sheibani V (2015) Exercise improves learning and memory impairments in sleep deprived female rats. Physiol Behav 138:285-291. https://doi.org/10.1016/j.physb eh.2014.10.006

11. Laurin D, Verreault R, Lindsay J, MacPherson K, Rockwood K (2001) Physical activity and risk of cognitive impairment and dementia in elderly persons. Arch Neurol 58(3):498-504

12. Maloyan A, Gulick J, Glabe CG, Kayed R, Robbins J (2007) Exercise reverses preamyloid oligomer and prolongs survival in alphaB-crystallin-based desmin-related cardiomyopathy. Proc Natl Acad Sci USA 104(14):5995-6000. https://doi.org/10.1073/ pnas.0609202104

13. Dvorakova-Lorenzova A, Suchanek P, Havel PJ, Stavek P, Karasova L, Valenta Z, Tintera J, Poledne R (2006) The decrease in $\mathrm{C}$-reactive protein concentration after diet and physical activity induced weight reduction is associated with changes in plasma lipids, but not interleukin-6 or adiponectin. Metabolism 55(3):359-365. https://doi.org/10.1016/j.metabol.2005.09.010

14. Chen YW, Li YT, Chen YC, Li ZY, Hung CH (2012) Exercise training attenuates neuropathic pain and cytokine expression after chronic constriction injury of rat sciatic nerve. Anesth Analg 114(6):1330-1337. https://doi.org/10.1213/ANE.0b013e3182 $4 \mathrm{c} 4 \mathrm{ed} 4$

15. Ji LL (2002) Exercise-induced modulation of antioxidant defense. Ann N Y Acad Sci 959:82-92

16. Ji LL, Gomez-Cabrera MC, Vina J (2006) Exercise and hormesis: activation of cellular antioxidant signaling pathway. Ann N Y Acad Sci 1067:425-435. https://doi.org/10.1196/annals.1354.061

17. Elsner VR, Basso C, Bertoldi K, de Meireles LC, Cechinel LR, Siqueira IR (2017) Differential effect of treadmill exercise on histone deacetylase activity in rat striatum at different stages of development. J Physiol Sci 67(3):387-394. https://doi. org/10.1007/s12576-016-0471-2

18. Radak Z, Chung HY, Koltai E, Taylor AW, Goto S (2008) Exercise, oxidative stress and hormesis. Ageing Res Rev 7(1):34-42. https://doi.org/10.1016/j.arr.2007.04.004

19. Halson SL, Jeukendrup AE (2004) Does overtraining exist? An analysis of overreaching and overtraining research. Sports Med 34(14):967-981

20. Gholamnezhad Z, Boskabady MH, Hosseini M, Sankian M, Khajavi Rad A (2014) Evaluation of immune response after moderate and overtraining exercise in Wistar rat. Iran J Basic Med Sci $17(1): 1-8$

21. Smith LL (2000) Cytokine hypothesis of overtraining: a physiological adaptation to excessive stress? Med Sci Sports Exerc 32(2):317-331

22. Sun LN, Li XL, Wang F, Zhang J, Wang DD, Yuan L, Wu MN, Wang ZJ, Qi JS (2017) High-intensity treadmill running impairs cognitive behavior and hippocampal synaptic plasticity of rats via activation of inflammatory response. J Neurosci Res 95(8):16111620. https://doi.org/10.1002/jnr.23996
23. Nakajima T, Kurano M, Hasegawa T, Takano H, Iida H, Yasuda T, Fukuda T, Madarame H, Uno K, Meguro K, Shiga T, Sagara M, Nagata T, Maemura K, Hirata Y, Yamasoba T, Nagai R (2010) Pentraxin 3 and high-sensitive $\mathrm{C}$-reactive protein are independent inflammatory markers released during high-intensity exercise. Eur J Appl Physiol 110(5):905-913. https://doi.org/10.1007/s0042 1-010-1572-x

24. Belviranlı M, Gökbel H (2006) Acute exercise induced oxidative stress and antioxidant changes. Euro J Gen Med 3(3):126-131. https://doi.org/10.29333/ejgm/82392

25. Clark A, Mach N (2016) Exercise-induced stress behavior, gutmicrobiota-brain axis and diet: a systematic review for athletes. J Int Soc Sports Nutr 13:43-64. https://doi.org/10.1186/s1297 0-016-0155-6

26. Kim H, Shin MS, Kim SS, Lim BV, Kim HB, Kim YP, Chung JH, Kim EH, Kim CJ (2003) Modulation of immune responses by treadmill exercise in Sprague-Dawley rats. J Sports Med Phys Fit 43(1):99-104

27. Hohl R, Ferraresso RL, De Oliveira RB, Lucco R, Brenzikofer R, De Macedo DV (2009) Development and characterization of an overtraining animal model. Med Sci Sports Exerc 41(5):11551163. https://doi.org/10.1249/MSS.0b013e318191259c

28. Vafaee F, Hosseini M, Sadeghinia HR, Hadjzadeh MA, Soukhtanloo M, Rahimi M (2014) The effects of soy extract on spatial learning and memory damage induced by global ischemia in ovariectomised rats. Malays J Med Sci 21(3):19-30

29. Anaeigoudari A, Shafei MN, Soukhtanloo M, Sadeghnia HR, Reisi P, Beheshti F, Mohebbati R, Mousavi SM, Hosseini M (2015) Lipopolysaccharide-induced memory impairment in rats is preventable using 7-nitroindazole. Arq Neuropsiquiatr 73(9):784790. https://doi.org/10.1590/0004-282x20150121

30. Kaveh M, Eidi A, Nemati A, Boskabady MH (2017) The extract of Portulaca oleracea and its constituent, alpha linolenic acid affects serum oxidant levels and inflammatory cells in sensitized rats. Iran J Allergy Asthma Immunol 16(3):256-270

31. Habeeb AF (1972) [37] Reaction of protein sulfhydryl groups with Ellman's reagent. Methods Enzymol 25:457-464. https:// doi.org/10.1016/S0076-6879(72)25041-8

32. Madesh M, Balasubramanian KA (1997) A microtiter plate assay for superoxide using MTT reduction method. Indian J Biochem Biophys 34(6):535-539

33. Aebi H (1984) Catalase in vitro. Methods Enzymol 105:121-126

34. Hosseini M, Harandizadeh F, Niazmand S, Soukhtanloo M, Faizpour A, Ghasemabady M (2014) The role for nitric oxide on the effects of hydroalcoholic extract of Achillea wilhelmsii on seizure. Avicenna J Phytomed 4(4):251-259

35. Gholamnezhad Z, Boskabady MH, Hosseini M, Aghaei A (2019) Effect of different loads of exercise and Nigella sativa L. seed extract on serologic and hematologic parameters in rat. Indian $\mathbf{J}$ Exp Biol 57:21-29

36. Lira FS, Rosa JC, Pimentel GD, Tarini VA, Arida RM, Faloppa F, Alves ES, do Nascimento CO, Oyama LM, Seelaender M, de Mello MT, Santos RV (2010) Inflammation and adipose tissue: effects of progressive load training in rats. Lipids Health Dis 9:109-119. https://doi.org/10.1186/1476-511X-9-109

37. Jahangiri Z, Gholamnezhad Z, Hosseini M (2018) Neuroprotective effects of exercise in rodent models of memory deficit and Alzheimer's. Metab Brain Dis 34:21-37. https://doi.org/10.1007/ s11011-018-0343-y

38. Reisi P, Alaei H, Babri S, Sharifi MR, Mohaddes G (2009) Effects of treadmill running on spatial learning and memory in streptozotocin-induced diabetic rats. Neurosci Lett 455(2):79-83. https ://doi.org/10.1016/j.neulet.2009.03.052

39. Wang XQ, Wang GW (2016) Effects of treadmill exercise intensity on spatial working memory and long-term memory in rats. Life Sci 149:96-103. https://doi.org/10.1016/j.1fs.2016.02.070 
40. Kim K, Sung YH, Seo JH, Lee SW, Lim BV, Lee CY, Chung YR (2015) Effects of treadmill exercise-intensity on short-term memory in the rats born of the lipopolysaccharide-exposed maternal rats. J Exercise Rehabil 11(6):296-302. https://doi.org/10.12965 /jer. 150264

41. Kennard JA, Woodruff-Pak DS (2012) A comparison of lowand high-impact forced exercise: effects of training paradigm on learning and memory. Physiol Behav 106(4):423-427. https://doi. org/10.1016/j.physbeh.2012.02.023

42. Ogonovszky H, Berkes I, Kumagai S, Kaneko T, Tahara S, Goto S, Radak Z (2005) The effects of moderate-, strenuous- and overtraining on oxidative stress markers, DNA repair, and memory, in rat brain. Neurochem Int 46(8):635-640. https://doi.org/10.1016/j. neuint.2005.02.009

43. Wu CW, Chen YC, Yu L, Chen HI, Jen CJ, Huang AM, Tsai HJ, Chang YT, Kuo YM (2007) Treadmill exercise counteracts the suppressive effects of peripheral lipopolysaccharide on hippocampal neurogenesis and learning and memory. J Neurochem 103(6):2471-2481. https://doi.org/10.111 1/j.1471-4159.2007.04987.x

44. Fehrenbach E, Niess AM, Voelker K, Northoff H, Mooren FC (2005) Exercise intensity and duration affect blood soluble HSP72. Int J Sports Med 26(7):552-557. https://doi. org/10.1055/s-2004-830334

45. Yirmiya R, Winocur G, Goshen I (2002) Brain interleukin-1 is involved in spatial memory and passive avoidance conditioning. Neurobiol Learn Mem 78(2):379-389

46. Palin K, Verrier D, Tridon V, Hurst J, Perry VH, Dantzer R, Lestage $J$ (2004) Influence of the course of brain inflammation on the endogenous IL-1beta/IL-1Ra balance in the model of brain delayed-type hypersensitivity response to bacillus CalmetteGuerin in Lewis rats. J Neuroimmunol 149(1-2):22-30. https:// doi.org/10.1016/j.jneuroim.2003.12.005

47. Rai S, Kamat PK, Nath C, Shukla R (2014) Glial activation and post-synaptic neurotoxicity: the key events in Streptozotocin (ICV) induced memory impairment in rats. Pharmacol Biochem Behav 117:104-117. https://doi.org/10.1016/j.pbb.2013.11.035

48. Baker JS, Bailey DM, Hullin D, Young I, Davies B (2004) Metabolic implications of resistive force selection for oxidative stress and markers of muscle damage during $30 \mathrm{~s}$ of high-intensity exercise. Eur J Appl Physiol 92(3):321-327. https://doi.org/10.1007/ s00421-004-1090-9

49. Nair A, Bonneau RH (2006) Stress-induced elevation of glucocorticoids increases microglia proliferation through NMDA receptor activation. J Neuroimmunol 171(1-2):72-85. https:// doi.org/10.1016/j.jneuroim.2005.09.012

50. Sugama S, Fujita M, Hashimoto M, Conti B (2007) Stress induced morphological microglial activation in the rodent brain: involvement of interleukin-18. Neuroscience 146(3):1388-1399. https://doi.org/10.1016/j.neuroscience.2007.02.043

51. Nakagawa Y, Chiba K (2014) Role of microglial $\mathrm{m} 1 / \mathrm{m} 2$ polarization in relapse and remission of psychiatric disorders and diseases. Pharmaceuticals (Basel, Switzerland) 7(12):1028-1048. https:// doi.org/10.3390/ph7121028

52. Moghaddasi M, Javanmard SH, Reisi P, Tajadini M, Taati M (2014) The effect of regular exercise on antioxidant enzyme activities and lipid peroxidation levels in both hippocampi after occluding one carotid in rat. J Physiol Sci 64(5):325-332. https:// doi.org/10.1007/s12576-014-0322-y

53. Radak Z, Zhao Z, Koltai E, Ohno H, Atalay M (2013) Oxygen consumption and usage during physical exercise: the balance between oxidative stress and ROS-dependent adaptive signaling. Antioxid Redox Signal 18(10):1208-1246. https://doi. org/10.1089/ars.2011.4498

54. Yu F, Xu B, Song C, Ji L, Zhang X (2013) Treadmill exercise slows cognitive deficits in aging rats by antioxidation and inhibition of amyloid production. NeuroReport 24(6):342-347. https:// doi.org/10.1097/WNR.0b013e3283606c5e

55. Hoffman JR, Im J, Kang J, Maresh CM, Kraemer WJ, French D, Nioka S, Kime R, Rundell KW, Ratamess NA, Faigenbaum AD, Chance B (2007) Comparison of low- and high-intensity resistance exercise on lipid peroxidation: role of muscle oxygenation. J Strength Cond Res 21(1):118-122. https://doi. org/10.1519/R-20526.1

56. Cunningham P, Geary M, Harper R, Pendleton A, Stover S (2005) High intensity sprint training reduces lipid peroxidation in fasttwitch skeletal muscle. J Exerc Physiol Online 8(6):18-25

57. Robertson J, Maughan R, Duthie G, Morrice P (1991) Increased blood antioxidant systems of runners in response to training load. Clin Sci 80(6):611-618

58. Aguiar AS Jr, Tuon T, Pinho CA, Silva LA, Andreazza AC, Kapczinski F, Quevedo J, Streck EL, Pinho RA (2008) Intense exercise induces mitochondrial dysfunction in mice brain. Neurochem Res 33(1):51-58. https://doi.org/10.1007/s11064-007-9406-x

59. Jahangiri Z, Gholamnezhad Z, Hosseini M (2019) The effects of exercise on hippocampal inflammatory cytokine levels, brain oxidative stress markers and memory impairments induced by lipopolysaccharide in rats. Metab Brain Dis. https://doi. org/10.1007/s11011-019-00410-7

Publisher's Note Springer Nature remains neutral with regard to jurisdictional claims in published maps and institutional affiliations. 